\title{
Few-Mode Fibre Group-Delays with Intermediate Coupling
}

\author{
Filipe M. Ferreira, Naoise Mac Suibhne, Stylianos Sygletos, Andrew D. Ellis \\ Aston Institute of Photonic Technologies, Aston University, Birmingham, UK (․fferreira@aston.ac.uk)
}

\begin{abstract}
We study the probability density function of the group-delay in few-mode fibres, validating for the first time an analytical estimation for the maximum group-delay spread as a function of linear mode coupling for fibres with more than three LP modes.
\end{abstract}

\section{Introduction}

Mode-division multiplexing (MDM) over fewmode fibres (FMFs) has been proposed as a promising candidate to overcome the capacity limit of single-mode fibres (SMFs). However, MDM-FMF systems require significantly higher equalizer complexity given the overall groupdelay (GD) spread due to differential mode delay and linear mode coupling ${ }^{1,2}$. Thereby, the estimation of the maximum GD spread is of foremost importance. However, the statistical properties of the GDs are only well known for the two extreme coupling regimes, weak and strong $^{1,2}$. Recently, we validated one single expression linking the standard deviation of GDs to the coupling strength for any coupling regime ${ }^{3}$, considering a FMF guiding 3 linearly polarized (LP) modes $\left(\mathrm{LP}_{01}, \mathrm{LP}_{11 \mathrm{a}}, \mathrm{LP}_{11 \mathrm{~b}}\right)$. However, to accurately estimate the maximum GD spread with a given confidence level the probability density function of the GD has to be studied.

In this paper, we extend our previous study ${ }^{3}$ to more than 3 LP modes and investigate for the first time the probability density function (PDF) of GD in FMFs for the intermediate coupling regime. Furthermore, we validate, for the first time, an analytical estimation for the maximum GD spread as a function of the coupling strength.

\section{Group-Delay Statistics}

In previous work ${ }^{4}$, it has been shown that the approach of considering principal states of polarization (PSPs) with well-defined GDs in SMFs, can be extended to FMFs. In FMFs, the coupled modes having well defined GDs were called principal modes (PMs). In both cases the statistics of the GDs are dependent on the linear coupling strength, which can be defined as a function of the correlation length $L_{c}$. In the SMF case, $L_{c}$ is defined as the length for which the average power lost to the orthogonal polarization is within $e^{-2}$ of the initial power ${ }^{5}$. In the FMF case, we generalize $L_{c}$ as the length for which $\left[P_{m}\left(L_{c}\right)-\sum_{v \neq m} P_{v}\left(L_{c}\right)\right] / \sum_{n} P_{n}\left(L_{c}\right)=e^{-2}$, where $P_{v}$ is the power of mode $v$ and mode $m$ is the mode presenting higher coupling strength. The coupling regimes may then be broadly defined as: strong coupling when $L>>L_{c}$, weak coupling when $L \ll L_{c}$, and intermediate coupling otherwise. In the FMF case, the statistical properties of the GDs are only well known for the two extreme regimes ${ }^{1,2}$. In those regimes, the standard deviation of the coupled GDs of the output PMs $\left(\sigma_{g d}\right)$ can be written as a function of the uncoupled GDs along one section $\left(\sigma_{\tau, \mathrm{sec}}\right)$. Assuming $K$ fibre sections with identical statistical properties, it has been shown that ${ }^{1,2}$ : in the weak coupling regime $\sigma_{g d}=K \sigma_{\tau, \text { sec }}$ and in the strong coupling regime $\sigma_{g d}=K^{1 / 2} \sigma_{\tau, \text { sec }}$. In both cases, the $\sigma_{g d}$ functional form is valid for any number of modes. Furthermore, the PDF of GDs has been derived analytically for strong coupling ${ }^{1,2}$.

In the intermediate coupling regime, the statistics of the GDs are not yet fully understood. In this regime, we have shown that at least for fibres guiding 3 LP modes, the standard deviation of the GDs in $\mathrm{SMFs}^{5}$ can be extended to $\mathrm{FMFs}^{3}$ :

$$
\sigma_{g d}=\sqrt{2} \sigma_{\tau} L_{c}\left(e^{-L / L_{c}}+\frac{L}{L_{c}}-1\right)^{1 / 2}
$$

where $\sigma_{\tau}$ is the standard deviation of the uncoupled GDs per unit length. In this paper, we will show that Eq. (1) can be applied to fibres guiding $6 \mathrm{LP}$ modes $\left(\mathrm{LP}_{01}, \mathrm{LP}_{11 \mathrm{a}}, \mathrm{LP}_{11 \mathrm{~b}}, \mathrm{LP}_{02}\right.$, $\left.\mathrm{LP}_{21 a}, \quad L P_{21 b}\right)$ despite the different coupling strengths between different pairs of modes belonging to different mode groups.

\section{Linear Mode Coupling}

To verify the applicability of Eq. (1), we use a numerical mode coupling model which divides the fibre in multiple sections of length $L_{s}$, each with a constant random displacement of the core centre position ${ }^{6}$. The model considers a random displacement of the radial and azimuthal coordinates. The mode coupling strength $(X T)$ is quantified as $X T_{m}=\sum_{n \neq m} P_{n} / P_{m}$ where $P_{n}$ is the power of mode $n$, after a given segment under test, when only mode $m$ was launched. If $X T_{m}$ is equal to $0 \mathrm{~dB}$, then half of the power launched in mode $m$ has been transferred to other modes.

Fig. 1 shows the mode coupling strength averaged over the azimuth displacement, as a function of the normalised radial displacement, for a 6 LP mode fibre. Note that, coupling 


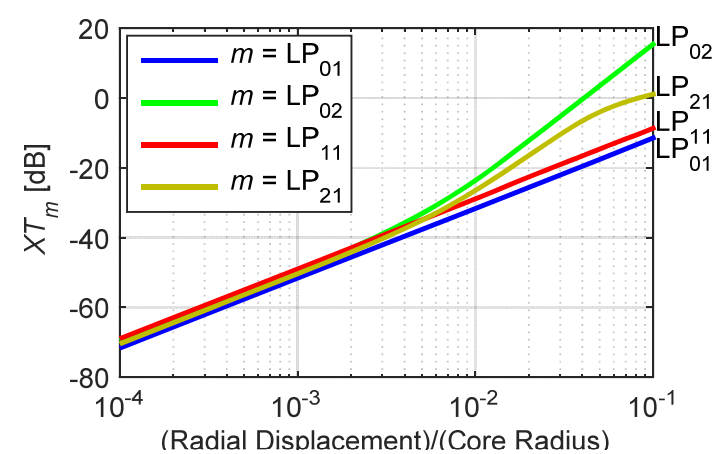

Fig. 1: $X T_{m}$ averaged over the azimuth displacement as a function of the radial displacement

strengths are calculated considering degenerate modes such as $L P_{11 a}$ and $L P_{11 b}$ as one mode.

In Fig. 1, it can be seen that the mode coupling strength only depends significantly on the mode being considered for displacements higher than $1 \%$. Such higher coupling for $\mathrm{LP}_{02}$ and $\mathrm{LP}_{21}$ can be explained noting they belong to the same LP mode group. Moreover, $X T_{L P_{21}}<X T_{L P_{02}}$ for any displacement in Fig. 1 because any power launched in $\mathrm{LP}_{21 a}$ couples preferentially with $\mathrm{LP}_{21 \mathrm{~b}}$ (and vice-versa) and in the second place to $\mathrm{LP} 02$. Given the higher values of $X T_{L P_{02}}$, we define $L_{c}$ for this mode. Note that $X T_{m}$ values above $10 \mathrm{~dB}$ mean that almost all power launched in mode $m$ has been transferred to other modes.

In the literature, the mode coupling values of fabricated FMFs range from $-50 \mathrm{~dB} / 100 \mathrm{~m}$ to $-40 \mathrm{~dB} / 100 \mathrm{~m}$ for fibres with step-index or graded-index profiles ${ }^{7,8}$, going up to $-28 \mathrm{~dB} / 100 \mathrm{~m}$ for coupled multi-core fibres ${ }^{9}$ and $-7 \mathrm{~dB} / 100 \mathrm{~m}$ for fibres with ring-index profiles ${ }^{10}$. In the next section, the impact of these coupling values on the GD spread is accessed.

\section{Results}

In this section, we evaluate the statistics of the GDs of the PMs. The FMF considered guides 6 modes and presents a DMD of $5.19 \mathrm{ps} / \mathrm{km}$ (we assumed zero DMD between degenerate LP modes) $)^{11}$. The $X T_{L P_{02}}$ value was varied from -50 to $0 \mathrm{~dB} / 100 \mathrm{~m}$ by varying the amplitude of the variation in lateral section offset, assuming a section length of $100 \mathrm{~m}$. This range fully covers the range of coupling values presented in the literature ${ }^{7-10}$. Finally, the GDs of the PMs were calculated through direct numerical solution of the coupled-mode equations describing the linear mode propagation ${ }^{6}$.

Fig. 2 shows the standard deviation of the GDs of the PMs $\left(\sigma_{g d}\right)$ as a function of distance up to $10,000 \mathrm{~km}$, obtained by averaging over 2000 different realizations of lateral offsets giving rise to a given $X T_{L P_{02}}$ value. As was found for $3 \mathrm{LP}$

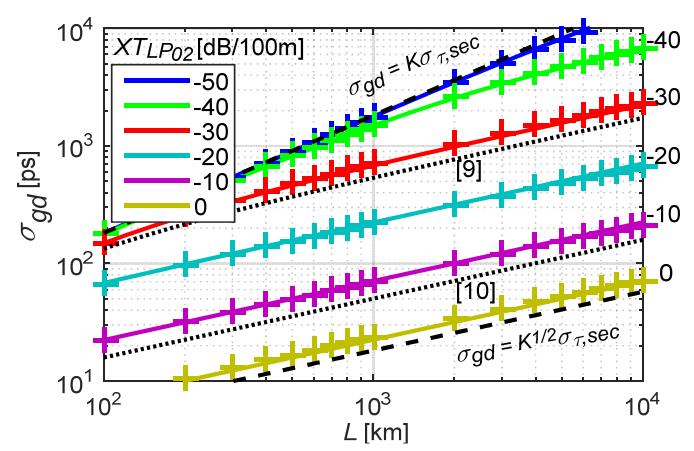

Fig. 2: Standard deviation of the GDs of the PMs as a function of transmission distance showing simulation results (markers) and analytical results (solid lines).

modes $^{3}$, Fig. 2 shows a good agreement between numerical simulation and the analytical expression deduced from the 2-mode approximation Eq. (1), for any coupling value studied and for any distance up to $10,000 \mathrm{~km}$. This verifies that Eq. (1) remains valid for 6-mode fibres (the validity for 3-mode fibres had been studied $\mathrm{in}^{3}$ ). In Fig. 2, it can be seen that for coupling values ranging from -50 to $-40 \mathrm{~dB} / 100 \mathrm{~m}$, $\sigma_{g d}$ scales approximately linearly with distance. But, at $-40 \mathrm{~dB} / 100 \mathrm{~m}$ the deviation from linear growth is already noticeable above $1,000 \mathrm{~km}$, thus even with such a low coupling, the FMF is operating in intermediate coupling regime. Increasing $X T_{L P_{02}}, \sigma_{g d}$ gradually converges to the strong coupling regime. However, even for a $X T_{L P_{02}}$ equal to $-7.01 \mathrm{~dB} / 100 \mathrm{~m}$ (the highest value found in literature ${ }^{10}$ ) the fibre is still not fully operating in the strong coupling regime when considering a section length of $100 \mathrm{~m}$. In this case, assuming strong coupling regime (random unitary matrices every $100 \mathrm{~m}$ ), would underestimated $\sigma_{g d}$ by a factor of 2.76.

Fig. 3 shows the PDF of the ordered GDs $\left(\tau_{m}\right.$, $\left.\tau_{1} \leq \tau_{2} \leq \cdots \leq \tau_{6}\right)$, normalized by the $\sigma_{g d}$ of the PMs, after $1000 \mathrm{~km}$ for two different coupling values, overlapped with the analytical joint PDF (thin black line) derived for the strong coupling regime $^{1}$. Note that the normalization factor $\left(\sigma_{g d}\right)$ depends on the $X T_{L P_{02}}\left(L_{c}\right)$ value, see Eq. (1). Fig. 3 (a) shows that for $-30 \mathrm{~dB} / 100 \mathrm{~m}$ the GDs of the PMs vaguelly resemble the GDs of the LP modes given the impulse-like PDF of $\tau_{2}$ ("LP11a") and $\tau_{3}$ ("LP $11 \mathrm{~b}$ "). Further results for lower coupling values shown that all GDs present impulse-like PDFs. In Fig. 3 (b), for $-20 \mathrm{~dB} / 100 \mathrm{~m}$, the match between the simulated PDFs and the analytical PDF for strong coupling is good, even though the GDs have been normalized by different factors (Eq. (1)). Further results shown that the match between the simulated PDFs and the analytical PDF improves for higher coupling values. A similar match was obtained for 3 modes. 


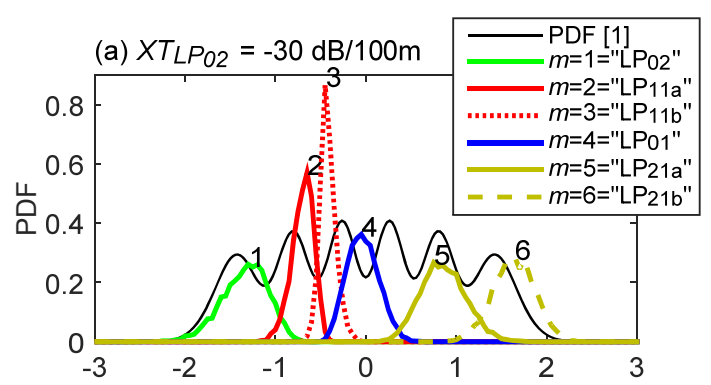

(b) $X T_{L P_{02}}=-20 \mathrm{~dB} / 100 \mathrm{~m}$

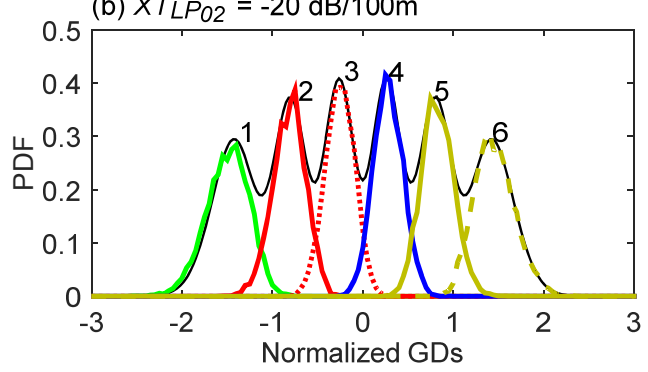

Fig. 3: Probability density function of the ordered normalized GDs $\left(\tau_{m} / \sigma_{g d}\right)$, obtained through simulation after $1000 \mathrm{~km}$, with different $X T_{L P_{02}}$ values.

In a MDM system, in order to fully compensate for DMD and mode coupling, the MIMO equalizer must span a temporal memory at least as long as the system GD spread $\left(\tau_{6}-\tau_{1}\right)$. Fig. 4 shows the complementary cumulative distribution function (CCDF) of the normalized GD spread, $\operatorname{Pr}\left(\left(\tau_{6}-\tau_{1}\right) / \sigma_{g d}>p\right)$, obtained through simulation after $1000 \mathrm{~km}$ for different coupling values (averaging over 6000 different realizations). Fig. 4 shows that for $X T_{L P_{02}} \geq-30 \mathrm{~dB} / 100 \mathrm{~m}$ the CCDFs are very similar to the analytical approximation obtained for strong coupling ${ }^{12}$ (dashed line). Conversely, for $X T_{L P_{02}}$ lower than $-30 \mathrm{~dB} / 100 \mathrm{~m}$ the normalized GD spread is significantly smaller than the normalized GD spread for strong coupling.

Combining this observation with the normalisation factor (Eq. (1)), we find that the required temporal equalizer memory length $\left(\Delta T_{E Q}\right)$ to span the channel memory with a given probability $p$ and for a given mode coupling strength is given by (in time units):

$\Delta T_{E Q} \cong U(p) \cdot \sigma_{g d}=U(p) \cdot \sqrt{2} \sigma_{\tau} L_{c}\left(e^{-L / L_{c}+\frac{L}{L_{c}}-1}\right)^{1 / 2}$

where $U(p)$ can be calculated using eq. 6 in [12], dashed line in Fig. 4. A $\Delta T_{E Q}$ from $4 \sigma_{g d}$ to $5 \sigma_{g d}$ is sufficient to span the channel memory with probability from $10^{-4}$ to $10^{-6}$.

\section{Conclusions}

In this paper, we reported an investigation on the statistics of the group-delays in FMFs. The investigation shows that even for the FMFs in the literature presenting high coupling strength $(-7 \mathrm{~dB} / 100 \mathrm{~m})$, the performance is not accurately

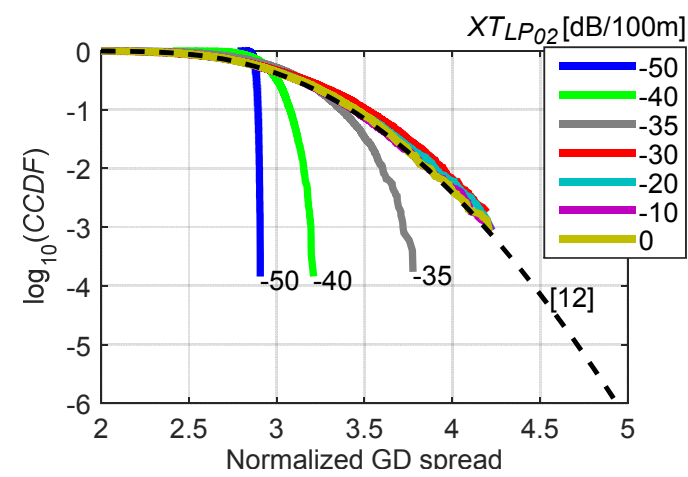

Fig. 4: Complementary cumulative distribution of the normalized GD spread, obtained through simulation

after $1000 \mathrm{~km}$, with different $X T_{L P_{02}}$ values.

modelled by random unitary matrices (every $\sim 100 \mathrm{~m}$ ), which give a factor of 2 error. Furthermore, for the first time, an analytical estimation for the maximum GD spread as a function of the coupling strength, was validated for FMFs guiding up to 6 LP modes and for any coupling regime. Therefore, this analytical estimaton is a valuable tool for the development of future FMF systems.

\section{Acknowledgements}

This work has been partially supported by the European Communities 7th Framework Program under grant agreement 619732 (INSPACE) and by the EPSRC under grant agreement EP/L000091/1 (PEACE).

\section{References}

[1] K. Ho, et al., "Statistics of group delays in multimode fiber with strong mode coupling," J. Lightw. Technol., Vol. 29, no. 21 , p. 3119 (2011).

[2] C. Antonelli, et al., "Stokes-space analysis of modal dispersion in fibers with multiple mode transmission," Opt. Express, Vol. 20, no. 11, p. 11718 (2012).

[3] F. Ferreira, et al., "Impact of Linear Mode Coupling on the Group Delay Spread in Few-Mode Fibers," Proc. OFC, Tu2D.1, Los Angeles (2015).

[4] S. Fan, et al., "Principal modes in multimode waveguides," Opt. Lett., Vol. 30, no. 2, p. 135 (2005).

[5] C. Poole, "Statistical treatment of polarization dispersion in single-mode fiber," Opt. Lett., Vol. 13, no. 8, p. 687 (1988).

[6] F. Ferreira, et al., "Nonlinear Semi-Analytical Model for Simulation of Few-Mode Fiber Transmission," Photon. Technol. Lett., Vol. 24, no. 4, p. 240 (2012).

[7] L. Grüner-Nielsen, et al., "Few Mode Transmission Fiber With Low DGD, Low Mode Coupling, and Low Loss," J. Lightw. Technol., Vol. 30, no. 23, p. 3693 (2012)

[8] T. Mori, et al., "Low DMD Four LP Mode Transmission Fiber for Wide-band WDM-MIMO System," Proc. OFC, OTh3K.1, Anaheim (2013).

[9] R. Ryf, et al., "Space-division multiplexed transmission over 4200-km 3-core microstructured fiber," Proc. OFC, PDP5C.2, Los Angeles (2012).

[10]N. Fontaine, et al., "Experimental investigation of crosstalk accumulation in a ring-core fiber," Proc. PSSTMS, TuC4.2, Waikoloa (2013).

[11]F. Ferreira, et al., "Design of Few-Mode Fibers with Mmodes and Low Differential Mode Delay," J. Lightw. Technol., Vol. 32, no. 3, p. 353 (2014).

[12]K. Ho, et al., "Delay-Spread Distribution for Multimode Fiber With Strong Mode Coupling," Photon. Technol. Lett., Vol. 24, no. 21, p. 1906 (2012). 